\title{
Development of comorbidity-adapted exercise protocols for patients with knee osteoarthritis
}

\author{
This article was published in the following Dove Press journal: \\ Clinical Interventions in Aging \\ 14 May 2014 \\ Number of times this article has been viewed
}

\author{
Mariëtte de Rooij' \\ Marike van der Leeden ${ }^{1,2}$ \\ Ellis Avezaat ${ }^{3}$ \\ Arja Häkkinen ${ }^{4}$ \\ Rob Klaver ${ }^{\prime}$ \\ Tjieu Maas ${ }^{5}$ \\ Wilfred F Peter' \\ Leo D Roorda' \\ Willem F Lems',6 \\ Joost Dekker ${ }^{1,7}$
}

'Amsterdam Rehabilitation Research Center, Reade, Amsterdam, the Netherlands; ${ }^{2} \mathrm{VU}$ University Medical Center Department of Rehabilitation Medicine, Amsterdam, the Netherlands; ${ }^{3}$ Sint Lucas Andreas Hospital, Department of Physical Therapy, Amsterdam, the Netherlands; ${ }^{4}$ Department of Health Sciences, University of Jyväskylä and Jyväskylä Central Hospital, Jyväskylä, Finland; ${ }^{5}$ HAN University, Institute of Health, GGM, Institute for Sports and Exercise Studies, Nijmegen, the Netherlands; ${ }^{6} \mathrm{VU}$ University Medical Center, Department of Rheumatology, Amsterdam, the Netherlands; ${ }^{7} \mathrm{VU}$ University Medical Center, Department of Psychiatry and Department of Rehabilitation Medicine, EMGO Institute,

Amsterdam, the Netherlands

Correspondence: Mariëtte de Rooij Amsterdam Rehabilitation Research Center, Reade, PO Box 5827I, 1040 HG Amsterdam, the Netherlands $\mathrm{Tel}+3 \mid 20589629$ |

Fax +3I 205896316

Email m.d.rooij@reade.nl
Background: Exercise therapy is generally recommended for patients with osteoarthritis (OA) of the knee. Comorbidity, which is highly prevalent in OA, may interfere with exercise therapy. To date, there is no evidence-based protocol for the treatment of patients with knee OA and comorbidity. Special protocols adapted to the comorbidity may facilitate the application of exercise therapy in patients with knee OA and one or more comorbidities.

Purpose: The purpose of this study was to develop comorbidity-adapted exercise protocols for patients with knee OA and comorbidity.

Method: Several steps were undertaken to develop comorbidity-adapted protocols: selection of highly prevalent comorbidities in OA, a literature search to identify restrictions and contraindications for exercise therapy for the various comorbid diseases, consultation of experts on each comorbid disease, and field testing of the protocol in eleven patients with knee OA and comorbidity.

Results: Based on literature and expert opinion, comorbidity-adapted protocols were developed for highly prevalent comorbidities in OA. Field testing showed that the protocols provided guidance in clinical decision making in both the diagnostic and the treatment phase. Because of overlap, the number of exercise protocols could be reduced to three: one for physiological adaptations (coronary disease, heart failure, hypertension, diabetes type 2, chronic obstructive pulmonary diseases, obesity), one for behavioral adaptations (chronic a-specific pain, nonspecific low back pain, depression), and one for environmental adaptations (visual or hearing impairments). Evaluation of patient outcome after treatment showed significant $(P<0.05)$ and clinically relevant improvements in activity limitations and pain.

Conclusion: Comorbidity-adapted exercise protocols for patients with knee OA were developed, providing guidance in clinical reasoning with regard to diagnostics and treatment. To evaluate the effectiveness of treatment in line with our protocols, a randomized clinical trial should be performed.

Keywords: knee osteoarthritis, exercise therapy, comorbidity, rehabilitation, arthritis, coexisting disease

\section{Introduction}

Osteoarthritis (OA) is among the diseases with the highest rates of comorbidity. ${ }^{1,2}$ Comorbidity can be defined as "any distinct additional clinical entity that has existed or that may occur during the clinical course of a patient who has the index disease under study". ${ }^{3}$ Common comorbidities in OA include cardiovascular diseases, diabetes, obesity, chronic obstructive pulmonary disease (COPD), chronic pain, depression, and visual and hearing impairments. ${ }^{4}$ Comorbidity in older adults with OA is associated with more pain, greater limitations in daily activities, and a worse prognosis with respect to these limitations. ${ }^{5,6}$ 
Performing exercises is one of the key recommendations in current guidelines for the management of knee $\mathrm{OA} ; ;^{7,8}$ this has been found to relieve pain and to reduce activity limitations. ${ }^{9}$ Comorbidity may interfere with the application of exercise therapy in $\mathrm{OA}$, however; ${ }^{10}$ for example, in persons with heart failure, only moderate-intensity resistance training is recommended, and the last repetitions should not be straining. ${ }^{11}$ Furthermore, the warming-up and cooling-down sessions should be prolonged; perceived exertion and/or dyspnea scales should take precedence over heart rate and work rate targets; and isometric exercises should be avoided. ${ }^{12}$

Because comorbidities have a significant influence on prognosis ${ }^{6}$ and may influence treatment, they should be routinely taken into account. ${ }^{13}$ Unfortunately, there is no evidence-based protocol available for the treatment of patients with knee OA and comorbidity. ${ }^{14}$ Current OA guidelines do not offer specific recommendations concerning comorbidity-associated exercise adaptations. ${ }^{7,8,15}$ It is often not feasible to combine different disease-specific treatment guidelines, since one treatment might interact negatively with another treatment or affect the natural course of a coexisting disease. ${ }^{16}$ Furthermore, in clinical practice, older adults with knee OA and (severe) comorbidity are seldom referred for exercise therapy; often drop out at an early stage of the treatment; or may be treated inadequately (eg, therapists may reduce the intensity of treatment to an ineffective level).

When dealing with comorbidity, a patient-centered rather than a disease-oriented approach, in which the process of decision making should be based on clinical reasoning, is preferred. ${ }^{16}$ The Hypothesis-Oriented Algorithm for Clinicians (HOAC) $\mathrm{II}^{17}$ describes a framework for clinical decision making in physical therapy; it addresses examination, evaluation, diagnosis, prognosis, and intervention in a specific patient. Although the HOAC II gives general direction in clinical reasoning, specific advice concerning comorbidityadapted OA exercise therapy and comorbidity is not available in the literature.

Therefore, there is a need for comorbidity-adapted protocols for exercise therapy in older adults with knee OA and comorbidity. These protocols are expected to improve the application of OA-specific exercise therapy, may help to avoid adverse events, and may improve the outcome of exercise therapy. The evaluation of complex interventions requires a phased approach, because of specific difficulties in developing, identifying, documenting, and reproducing the intervention. To design a complex intervention, we used the Medical Research Council (MRC) framework, which was developed to help researchers to define clearly where they are in the research process. ${ }^{18}$ The framework describes four phases in the design and evaluation of complex interventions: the preclinical or theoretical phase; Phase I, or the modeling phase; Phase II, or the exploratory trial; and Phase III, or definitive randomized controlled trial. In the preclinical or theoretical phase, the evidence that the intervention might have the desired effect is identified. The theoretical basis for the intervention is reviewed and potentially active ingredients are identified. In Phase I, or the modeling phase, the components of the intervention are defined and tested, using qualitative techniques (eg, case studies). In Phase II, or the exploratory trial, the optimum intervention is developed, based on the information gathered in Phase I. Phase III consists of the definitive randomized controlled trial, and Phase IV the long-term implementation of the intervention. ${ }^{18}$

In a previous study, restrictions and contraindications for exercise therapy for patients with knee OA and comorbidity (theoretical phase) were identified in the literature. ${ }^{10}$ The purpose of the present study was to develop comorbidityadapted exercise protocols for older adults with knee OA and comorbidity (Phase I, modeling phase).

\section{Methods \\ Development of comorbidity- adapted protocols}

Five steps were undertaken to develop comorbidity adapted protocols. First, based on previous work, ${ }^{4}$ we selected comorbidities in OA that 1) are common (present $>5 \%$ ), and 2) have impact on pain and/or affect daily functioning. The following comorbidities were selected: cardiac diseases; hypertension; type 2 diabetes; obesity; COPD; low back pain; chronic pain; depression; and visual or hearing impairments. ${ }^{4}$ Second, a literature search in the PubMed (publication date range 1966-2009) database was conducted to make an inventory of restrictions and contraindications for exercise therapy in patients with OA of the knee and highly prevalent comorbidities. The method and the results of this search have been reported previously. ${ }^{10}$ Third, a preliminary version of the protocols was developed. Based on the results of the first two steps, comorbidity-related adaptations to the diagnosis and treatment of $\mathrm{OA}$ were described. Guidelines on exercise therapy in each comorbidity (eg, cardiac disease, diabetes, COPD, and nonspecific low back pain) were consulted. ${ }^{19-23}$ If there was no exercise therapy guideline available for a specific comorbidity, an available medical guideline was used (eg, guidelines for depression or hypertension). ${ }^{24,25}$ The principles described in these guidelines were incorporated into the adapted protocols for 
exercise therapy in OA of the knee. Fourth, the preliminary versions of the protocols were discussed with clinical experts in the fields of each comorbid disease and, subsequently, based on their feedback, further improved. The experts had extensive experience in the fields of cardiac rehabilitation, diabetes, COPD rehabilitation, chronic nonspecific pain, and visual and hearing impairments. Advice was sought on the treatment of each comorbidity and on how the principles of exercise therapy and training of the comorbid diseases should be incorporated into the exercise regimen for OA of the knee. After optimizing the protocols, the clinical experts were consulted again for the collection of feedback and to gain final consensus on the protocols.

Fifth, the draft protocols were field-tested in a pilot study in patients with knee OA and the target comorbidities. Thereafter, the protocols were further improved, based on the feedback from therapists and patients, leading to a final version of the protocols. The method for field-testing of the protocols in this pilot study is further described below.

\section{Field-testing}

\section{Procedure}

Participants were referred to our rehabilitation center by their general practitioner because of persistent knee problems. Participants' eligibility was assessed by physical examination by a rheumatologist and a rehabilitation physician. Physical measurements were carried out by a research assistant and questionnaires were filled out by the participants. The questionnaires and physical tests were administered at baseline and directly after treatment. The study was approved by the Medical Ethical Review Board of the Slotervaart Hospital and Reade, Amsterdam, the Netherlands. All participants gave written informed consent and the study was conducted in accordance with the Handbook for Good Clinical Research Practice of the World Health Organization and Declaration of Helsinki principles. ${ }^{26}$

\section{Participants}

Fourteen participants were recruited. Inclusion criteria were: 1) diagnosis of knee OA according to the clinical American College of Rheumatology criteria; ${ }^{27}$ 2) presence of at least one of the target comorbidities, ie, coronary disease, heart failure, hypertension, type 2 diabetes, obesity, COPD, chronic pain, nonspecific low back pain, depression, and vision and/ or hearing impairment (diagnosed by a medical specialist); 3) severity score $\geq 2$ of the comorbidity on the Cumulative Illness Rating Scale, ${ }^{28}$ indicating that the comorbidity has an impact on daily activities; and 4) the primary treatment goal should be OA related (instead of comorbidity related). Exclusion criteria were: 1) indication for total knee replacement; 2) inability to participate in treatment, eg, due to transport problems; 3) insufficient capacity in the Dutch language.

\section{Therapists}

The protocols were applied and evaluated by three qualified physical therapists with extensive experience (3, 8, and 12 years) in knee/hip rehabilitation OA treatment. In addition, two of the three therapists were members of the Committee for Hip and Knee OA Guideline Development for the Royal Dutch Society of Physical Therapy.

\section{Measurements}

To evaluate the treatment process, the therapists completed a weekly registration form, providing information about the duration of the treatment period, content of the treatment, adaptations in the treatment due to the comorbidity, and any problems encountered in applying the protocols. Adverse events, defined as any undesirable experience occurring in a subject during the study (regardless of whether or not this was related to the treatment), were registered. To evaluate the feasibility of the protocols, semi-structured interviews were held by the first author (MdR) along with therapists and participants. Topic lists were used to structure the interview (see Table 1). To evaluate patient outcome after treatment, performance-based tests were performed and self-reported questionnaires were filled in by participants at baseline and directly after treatment.

Functional ability was assessed with self-reported questionnaires and performance-based measurements. The Western Ontario and McMaster Universities Osteoarthritis Index (WOMAC) is a disease-specific, self-administered questionnaire, developed to study patients with hip and knee OA. ${ }^{29,30}$ The WOMAC consists of 24 questions grouped into three subscales (pain: five questions; stiffness: two questions; and physical function: 17 questions) and scaled in a 5-point Likert (LK) scale. The maximum score in the LK scale is 20 points for pain, 8 points for stiffness, and 68 points for physical function. Higher scores indicate more pain, stiffness, or limitations. The WOMAC is widely used in clinical research, and has been shown to be reliable, valid, and responsive for use in patients with OA. ${ }^{29-31}$ The PatientSpecific Functioning Scale was used to evaluate limitations in activities of the individual patient. ${ }^{32}$ Patients were asked, "Which activities do you perceive as important and were hampered by knee pain during the last week?" A list of activity suggestions was offered to support recall, and patients 
Table I Brief summary of specific adaptations to osteoarthritis exercise therapy due to comorbid disease

Hypertension

Coronary disease/ heart failure
- Contraindications for participation in the training program include: resting systolic blood pressure of $>200 \mathrm{mmHg}$ or diastolic blood pressure of $>115 \mathrm{mmHg}$.

- Check blood pressure-lowering medication with physician. If adequate but still hypertensive, low-to-moderate intensity strength straining should be performed instead of high-intensity strength training.

- Be aware that medication to lower blood pressure, like beta blockers, can limit exercise tolerance in persons without myocardial ischemia.

- Contraindications for participation in the training program include: progressive increase in heart failure symptoms; severe ischemia of the cardiac muscle upon exertion; dyspnea while speaking; respiratory frequency of more than 30 breaths per minute; heart rate at rest $>110 \mathrm{bpm}, \mathrm{VO}_{2} \max <10 \mathrm{~mL} / \mathrm{kg} /$ minute; ventricular tachycardia upon increasing exertion; fever; acute systemic diseases; recent pulmonary embolism ( $<3$ months ago) causing severe hemodynamic strain; thrombophlebitis; acute pericarditis or myocarditis; hemodynamically serious aortic stenosis or mitral valve stenosis; presence of unstable angina, for example, pain in the chest at rest or pain that does not react to specific medication; NYHA functional classification class 4; myocardial infarction less than 3 months before the start of the training program; atrial fibrillation with rapid ventricular response at rest ( $>100 \mathrm{bpm})$; weight gain of $>2 \mathrm{~kg}$ within a few days, whether or not accompanied by increased dyspnea at rest is related to weight gain.

- Use the results of a maximum or symptom-limited exercise test to calculate the individual aerobic exercise intensity in patients with cardiac problems. (If the patient is using beta blockers, the exercises should be based on the results of the maximum or symptom-limited exercise test with beta blocker use). The optimized exercise zone can be calculated using the Karvonen formula, which calculates the exercise heart rate as a percentage of the heart rate reserve (the difference between the maximum heart rate and the heart rate at rest), added to the resting heart rate. Patients should start with 2 weeks of exercise at $40 \%-50 \%$ of their $\mathrm{VO}_{2}$ max then gradually raise the training intensity from $50 \%$ to $80 \%$ of their $\mathrm{VO}_{2} \max$ or $\mathrm{VO}_{2}$ reserve.

- Base the exercise intensity on a percentage of the maximum capacity expressed in watts or METs and/or a Borg RPE scale ${ }^{50}$ (6-20) if the patient's heart rate does not rise sufficiently during the maximum or symptom-limited exercise test.

- Prolong the warming-up and cooling-down sessions to decrease the risk of cardiac decompensation.

- Reduce the training intensity in warm climatic conditions.

- Terminate the exercise session in patients with coronary heart disease if any of the following signs of strain upon exertion apply: angina; impaired pump function (shortness of breath disproportionate to exertion: abnormal fatigue disproportionate to exertion, increased peripheral/central edema); arrhythmias (high heart rate not in proportion to exertion, irregular heartbeat, changes in known arrhythmias); abnormal increase or decrease of blood pressure; fainting; dizziness; vegetative reactions (eg, excessive perspiring, pallor).

- Terminate the exercise session in patients with heart failure if any of the following reasons for excessive strain apply: severe fatigue or dyspnea out of proportion to the level of exertion; increase in breathing rate out of proportion to the level of exertion; low pulse pressure $(<10 \mathrm{mmHg})$; reduction of systolic blood pressure during exercise $(>10 \mathrm{mmHg})$; increasing ventricular or supraventricular arrhythmias; angina; vegetative reactions such as dizziness or nausea.

- Avoid a rapid increase in the peripheral resistance training in patients with heart failure, as this increases the afterload strongly and the risk of decompensation. For improving muscle strength, start with 2 weeks on $30 \%-40 \%$ of IRM and then gradually increase the resistance from $50 \%$ to $70 \%-80 \%$ of IRM.

- Perform interval training for patients in poor physical condition instead of aerobic training.

Type 2 diabetes $\quad-$ In the case of insulin-dependent diabetes patients monitor blood glucose levels before and after the training and in the evening.

- Postpone exercise training in case of blood glucose values $\leq 5$ and $\geq 15 \mathrm{mmol} / \mathrm{L}$.

- Avoid intensive resistance training in type 2 diabetes patients with retinopathy grade $\geq 3$.

- Check patients with type 2 diabetes regularly for wounds and sensory defects (monofilaments).

- Be aware of autonomic neuropathy. This may result in decreased cardiovascular response to exercise, impaired response to dehydration, impaired thermoregulation due to impaired skin blood flow and sweating, postural hypotension, and/or decreased maximum aerobic capacity. The patient's heart rate may not rise or abate sufficiently during or after the training.

- Contraindications for participation in the training program include pneumonia and exceptional loss of bodyweight $(10 \%$ in the past half year or $>5 \%$ in the past month).

- Start with interval training in patients with COPD with ventilator limitation or disturbed oxygen transport in the lungs (hypoxemic [saturation $<90 \%$ ]/hypocapnic $\left[\mathrm{PaCO}_{2}>55 \mathrm{mmHg}\right.$ ] during exercising). Start endurance training if walking on $70 \%$ of maximum watts level for at least 10 minutes is possible.

- Check saturation level: patients with pulmonary problems should not desaturate; this usually means that $\mathrm{O}_{2}$ saturation $\left(\mathrm{SaO}_{2}\right)$ should remain $\geq 90 \%$ during exercising (and should not fall by $\geq 4 \%$ ).

- Give advice and exercises targeting body position and breathing if hyperinflation is present.

- Be aware of a poor nutritional status.

- Coach the patient when there is presence of fear of exercise due to breathlessness.

- Stimulate weight reduction due to overweight or obesity and/or refer to a dietician.

- Reduce weight-bearing exercises because of increase in knee joint pain.

- Reduce the training intensity in warm climatic conditions. 
Table I (Continued)

Chronic nonspecific pain/nonspecific low back pain/depression
- Contraindications for participation in the training program include serious psychiatric disorders, a major depression, or specific spinal pathology.

Provide a graded activity program. ${ }^{49}$

- Educational message: not pain relief, but improvement of functioning is the primary goal of the treatment. Exercise and physical activity are recommended. The performance of physical activity should not depend on the amount of pain.

- With patients, select problematic activities (maximum of three) from an activity list.

- Set short-term and long-term goals for each activity and record them in a treatment agreement form.

- Determine baseline values of the patient by performing the selected activities until (pain) tolerance over I week and record these activities in a diary.

- Determine the duration of the treatment program. An individually based scheme is made on a time-contingent basis for each activity and exercise, starting slightly under baseline values and increasing gradually towards the preset short-term goal. Patients should neither underperform nor overperform on this gradually increasing scheme. The exercise quotas are preset and not subject to change during the course of the intervention, regardless of level of pain.

- Use performance charts to record and visualize the performance of activities and exercises.

- Give positive reinforcement toward healthy and active behavior; pain behavior is ignored to extinguish the pain behavior.

- Coach patients on coping with stress and fear of movement.

- Interrupt the gradual increase of activities when an active inflammatory process is suspected or diagnosed (eg, redness of the knee, increase in knee effusion, or comparable symptoms). Hereafter, the increase of activities starts at a lower level. In case of recurrent inflammatory processes, the treatment goal needs to be changed and the rate of increasing activities needs to be decelerated.

- Adapt the starting position of exercises, reduce the training intensity, and advise the patient to stay active in case of acute/ subacute low back pain ( $<3$ months).

- Give the patient time to discuss feelings due to depression and avoid appointments early in the morning.

Hearing and/or visual impairments
- Change the way in which patients are handled and use more manual guidance in case of visual impairments.

- Check whether or not the patient has understood the information in case of hearing impairments.

- Change the training environment when possible, eg, take into account the lighting or background noise in the exercise hall, line-of-sight communication, or poor auditory impressions.

- Add balance training in case of poor balance in patients with hearing or visual impairments.

- Coach patients in order to reduce fear of falling.

- Use a sign-language interpreter if normally used by the patient.

- Be aware of orientation difficulties due to hearing of visual impairments.

- Adapt the font or size of the font in prescribed exercise instructions for those with impaired vision.

Abbreviations: IRM, one-repetition maximum; bpm, beats per minute; COPD, chronic obstructive pulmonary disease; MET, metabolic equivalent; NYHA, New York Heart Association; $\mathrm{PaCO}_{2}$, partial pressure of oxygen in the blood; $\mathrm{SaO}_{2}$, saturation level of oxygen in hemoglobin; $\mathrm{VO}_{2}$ max, maximal oxygen uptake.

were allowed to provide other limited activities that were not on the list. Of these activities, the patient selected three main activities and ranked them in order of importance. The difficulty of performance of the main activities were scored by self-assessment on a numeric rating scale ([NRS from 0-10] $0=$ no problems to perform the activity; $10=$ impossible to perform the activity). Patient-Specific Functional Scale is an efficient and valid measure for assessing limitations in activities and change in limitations in persons with knee dysfunction. ${ }^{32}$ The Get Up and Go test ${ }^{33,34}$ was performed with subjects seated on a high standard chair (seat height 49 $\mathrm{cm})$. The subjects were instructed to stand up without the help of the arms on the command "go" and walk $15 \mathrm{~m}$ along an unobstructed corridor as fast as possible without running. The chronometer was stopped when they reached the $15 \mathrm{~m}$ mark on the floor. All subjects wore walking shoes. Patients who normally used walking devices were allowed to use them during the test. A longer time taken to perform the test was considered a higher activity limitation. The 6-minute walk test was completed by patients on a $30 \mathrm{~m}$ walkway. Patients were instructed to walk their maximum distance in a 6-minute period. The total distance covered in meters during 6 minutes of walking was scored..$^{35,36}$

Quality of life was assessed with the 36-Item Short-Form Health Survey (SF-36). ${ }^{37}$ The SF-36 is a widely applied generic instrument for measuring health status and consists of eight dimensions: physical functioning, social functioning, physical role, emotional role, mental health, energy, pain and general health perception. The SF-36 gives scores on a $0-100$ scale, with higher scores indicating better health. The reliability (median reliability coefficient 0.85 for all subscales) for the SF-36 has been established, ${ }^{38-40}$ and its validity has been shown in an elderly population, in which the instrument distinguished between those with and without poor health. ${ }^{41}$

Psychological functioning was assessed with the Hospital Anxiety and Depression Scale (HADS). ${ }^{42}$ The HADS is a self-report rating scale of 14 items on a 4-point LK scale 
(range: 0-3). It is designed to measure anxiety and depression (seven items for each subscale). The total score is the sum of the 14 items, and, for each subscale, the score is the sum of the respective seven items (ranging from 0-21). The HADS is widely used in clinical research and has been shown to be reliable, valid, and responsive for use as a screening tool in patients with OA. ${ }^{43}$

Pain was assessed with a subscale of the WOMAC. Muscle strength was assessed for flexion and extension of the knee using an isokinetic dynamometer (EnKnee; EnrafNonius B.V., Rotterdam, the Netherlands). ${ }^{44}$ Quadriceps and hamstring strength were measured isokinetically at $60 \% \mathrm{sec}$ ond. Patients performed a maximum of three test repetitions to measure the strength of the quadriceps and hamstrings for each knee. Mean muscle strength per leg was calculated to obtain a measure of overall leg muscle strength (in Nm). Subsequently, mean muscle strength was divided by the patient's weight to control for the correlation between muscle strength and weight. This measure (in $\mathrm{Nm} / \mathrm{kg}$ ) was used for the analyses. Excellent intra-rater reliability (intraclass correlation coefficient 0.93 ) has been reported for this measure in knee OA patients. ${ }^{45}$

At the end of the treatment, patients were asked to rate global perceived effect (GPE) of the treatment ${ }^{46}$ on a scale of 1-9, with a score of 1 meaning much better, 5 meaning no change, and 9 meaning much worse. Patient satisfaction with the kind of treatment was measured by the NRS (0-10), with higher scores indicating greater satisfaction.
Additional data recorded were age, sex, and duration of complaints. The weight (kg) and height $(\mathrm{m})$ were measured in standing position. Body mass index was calculated using the standard formula $\left(\mathrm{kg} / \mathrm{m}^{2}\right)$. Comorbidity was scored with the Cumulative Illness Rating Scale. ${ }^{28}$ Radiographs of the knee were scored using the grading scales proposed by Kellgren and Lawrence $(\mathrm{K} \& \mathrm{~L}){ }^{47}$

\section{Analysis}

In order to evaluate the treatment process, a descriptive analysis of the treatment registration forms was performed. The feasibility of the protocols was evaluated by analyzing the notes that were taken during the interviews with the therapists and participants. A faithful depiction of the experiences of the participants and therapists was achieved by verifying with the participant or therapist whether the remarks were interpreted in a correct way by giving a summary at the end of the interview. To analyze the patient outcomes after treatment, change scores were determined by subtracting the baseline scores from the posttreatment scores. Because the data were not normally distributed, pre- and posttreatment scores were analyzed with a nonparametric Wilcoxon signed-rank test $(P$-value $<0.05)$.

\section{Results \\ Results of the development of the protocols}

Eleven draft protocols for exercise therapy in persons with knee OA and comorbidity were developed based on our literature search and consultation with experts. Regular OA

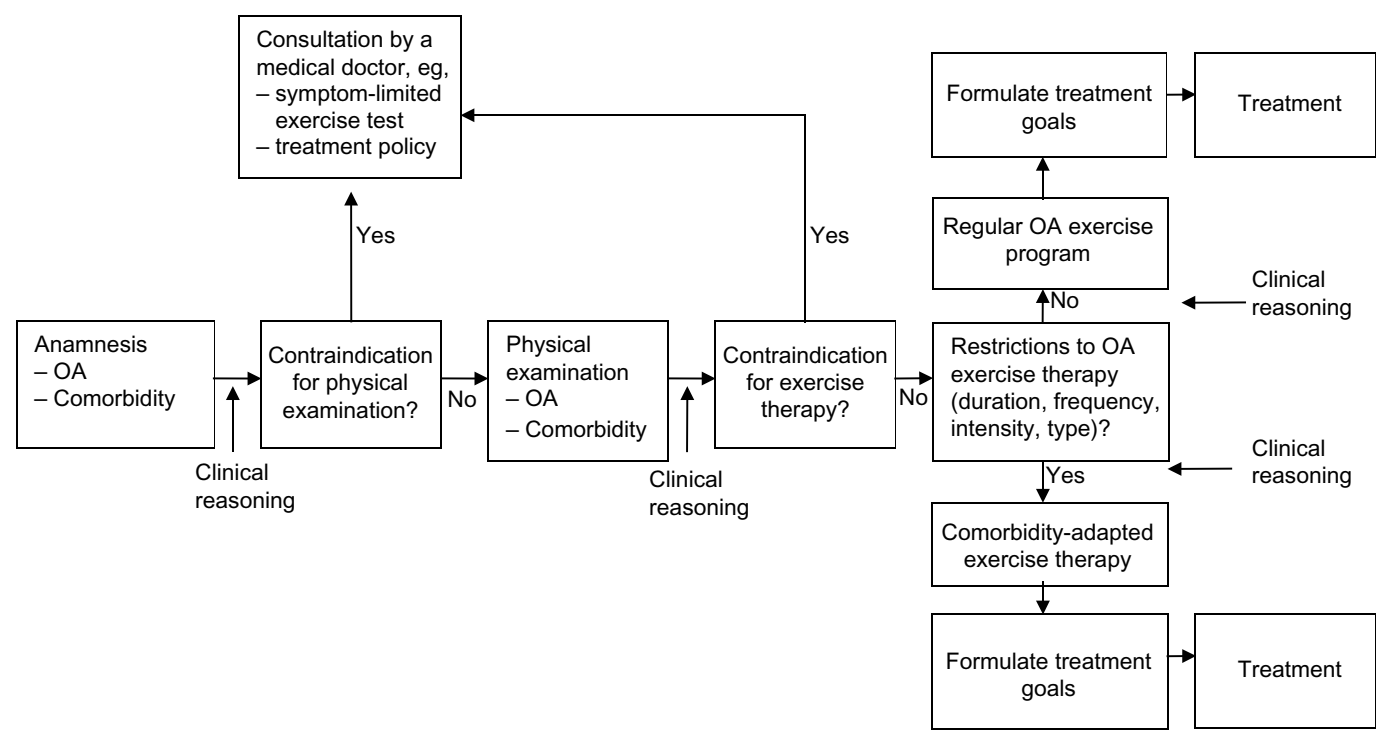

Figure I Flowchart of physical therapy intake.

Abbreviation: OA, osteoarthritis. 
exercise therapy as recommended in OA guidelines ${ }^{7,8,15}$ was the basis of the protocols. In the protocols, it was made explicit 1) how comorbidity compromises the regular application of exercise therapy in OA of the knee, and 2) how the therapist should consider the whole system, consisting of integrated body structures/functions and activities instead of separate organs, for all phases of treatment (examination, evaluation, diagnosis, prognosis, and intervention). The HOAC II framework was used to incorporate principles of clinical reasoning into the protocols. ${ }^{17}$

The protocols on exercise therapy in persons with knee $\mathrm{OA}$ and comorbidities consist of a diagnostic phase and an intervention phase. Each step in the protocols encourages clinical reasoning in order to tailor the diagnostic and intervention phase to the individual person. To facilitate this process, we designed a flowchart for the diagnostic and intervention phase (Figure 1).

The diagnostic phase includes an anamnesis, physical examination, establishment of treatment goals, and determination of the treatment strategy. During the anamnesis, OA-related problems, comorbidity-related restrictions and contraindications for exercise therapy are identified. Thereafter, a clinical decision is made as to whether physical examination is possible, or whether the referring physician needs to be consulted because of contraindications for physical examination or the need for further medical information. With respect to the latter, test results of a maximum symptom-limited exercise test may be required (for example, for persons with heart failure) to establish an appropriate training intensity.

If there are no contraindications for physical examination, comorbidity-related examination is performed according to the protocols (eg, foot examination in patients with type 2 diabetes). Subsequently, a decision is made as to whether there are contraindications or restrictions for exercise therapy. In case of a contraindication, referral to a physician is indicated. If there are comorbidity-related restrictions for exercise therapy, a comorbidity-adapted program is indicated. In this phase, the therapist also considers whether referral to professionals in other disciplines (eg, a dietician, psychologist, or occupational therapist) is indicated.

With regard to the intervention phase, the basic intervention in persons with knee OA consists of regular exercise therapy, according to the Royal Dutch Society for Physical Therapy's guideline for physical therapy in patients with knee $\mathrm{OA},{ }^{15}$ which is similar to international guidelines. ${ }^{7,8}$ Regular exercises for patients with OA comprise exercises aiming at improvement of muscle strength, aerobic capacity, flexibility, and ability to perform daily weight-bearing activities such as walking, stair climbing, and transfers (eg, sitting down or standing up from a chair). Individual therapy is given two times per week for between 30 to 60 minutes per session. The training intensity is increased from $40 \%-85 \%$ of the maximum oxygen uptake $\left(\mathrm{VO}_{2} \mathrm{max}\right)$ or the heart rate reserve. The increase of training intensity is monitored by using the Borg RPE scale (6-20) or heart rate frequency. The eventually obtained training intensity depends on the condition of the patient. Participants are encouraged to perform exercises at home at least five times per week. The treatment ends when treatment goals are achieved or when no further improvement is feasible.

The regular OA exercises are adapted to the comorbidity by changes in the duration, frequency, intensity, and type (content) of exercise therapy. The exact adaptations depend on restrictions for exercise therapy identified by the therapist in the diagnostic phase (anamnesis and physical examination). The specific options for adaptations to OA exercises are listed in the protocols and summarized in Table 1.

\section{Results from the field-testing}

Fourteen participants were included in the study. Three participants dropped out, one because of ocular problems due to diabetes and two others because of comorbidities not included in this study (hemochromatosis and cancer). Drop-out was unrelated to treatment. Nine of the eleven remaining participants had two or more comorbidities (Table 2).

Table 2 provides results of the evaluation of the treatment process. The duration of the treatment ranged from 14 to 20 weeks. The normal duration of the treatment in persons with knee OA without comorbidity in our center is 12 weeks. There were no adverse events reported during the study.

In participants with comorbidities resulting in physiological impairments (coronary disease, heart failure, type 2 diabetes, COPD, and obesity; $n=6$ ), four were referred back to the general practitioner or specialist because of a high or fluctuating blood pressure. In these cases, while medication was adjusted, aerobic and strength exercises were postponed during the first 4 to 6 weeks of treatment. The training intensity started at a low level and was gradually increased. Whole-body training or arm training was applied when loadability of the lower extremities was extremely low. This occurred mostly in participants with more than two comorbidities.

In participants with comorbidities resulting in behavioral impairments (chronic pain and nonspecific low back pain; $n=4$ ), adaptations were made by using a combined 


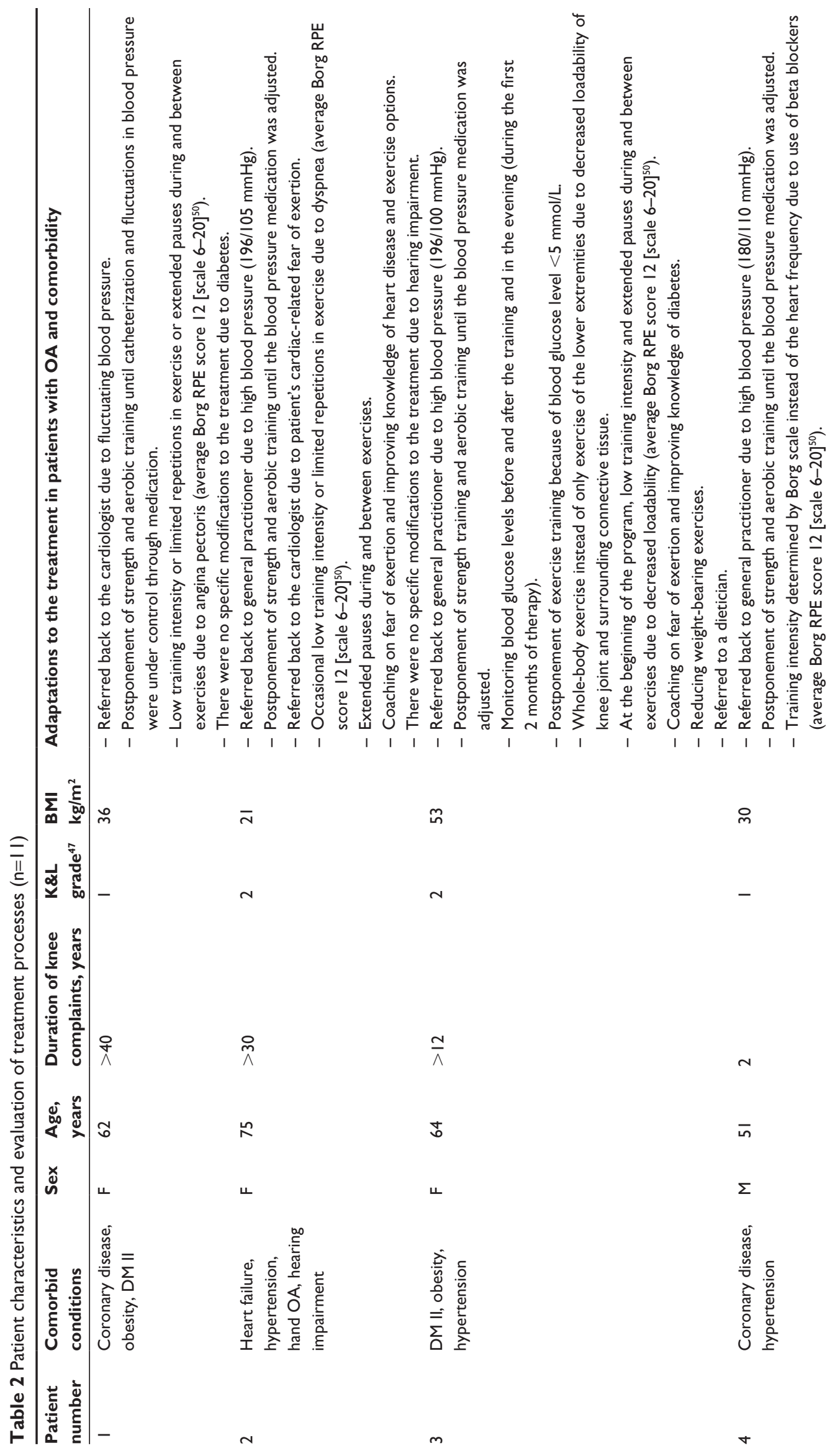




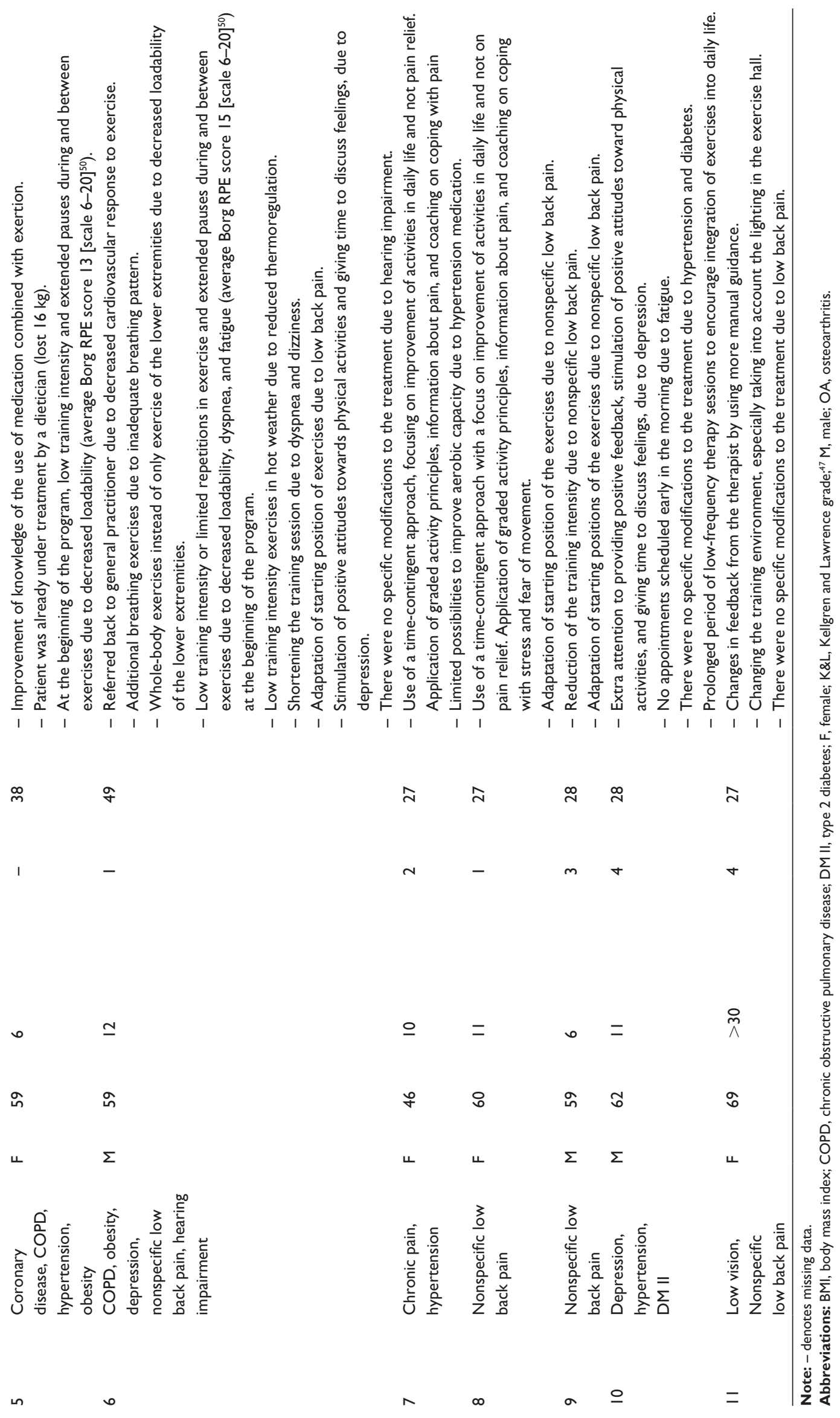


Table 3 Feasibility of the protocols

Topic
Was it possible to integrate the protocols when multiple comorbidities
were present?
Did you follow one primary protocol if multiple comorbidities were
present?
Is it possible to reduce the II protocols to fewer protocols?

Did the protocol help you in your clinical decision making process during the diagnostic and treatment phases? If so, in what way(s)?

Did you encounter any obstacles when providing the treatment? Do you have suggestions for improvements?

\section{Topic}

Were the patients satisfied with the treatment?

Were there any comorbidity-related problems during the treatment?

Was the duration of the diagnostic phase (too) intensive for the patient?

Did the patients have any suggestions to improve the protocol?

\section{Summary of therapists' answers}

Integration of the protocols was possible. If more than one comorbidity was present, more emphasis was placed on the protocol for the comorbidity with the highest impact on physical functioning. This could change over time because of changes in health status.

The II protocols can be reduced to three main protocols: a protocol for physiological related impairments, a protocol for behavioral related impairments and a protocol related to environmental impairments.

Reducing the number of protocols is expected to increase the feasibility. The protocol was helpful in clinical decision making and prevented exclusion from treatment due to lack of knowledge about the comorbidity or loadability of the patient. It was possible to tailor the exercise program to the individual capacity of the patient.

No specific obstacles were mentioned

Reduce overlap in the protocols in the diagnostic and treatment phase if more comorbidities are present.

\section{Summary of patients' answers}

Mean score on the NRS satisfaction (0-10) was 8 points (range 7-10). No specific problems were mentioned.

None of the patients experienced problems with the extended duration of the intake phase. Patients were satisfied with the attention to their health conditions, which gave them more confidence in performing exercises. One patient suggested planning a standard appointment with a social worker or psychologist in the intake phase.

Abbreviation: NRS, numeric rating scale.

behavioral approach with regular OA exercises. In a timecontingent manner, the amount of physical activity was gradually increased combined with a gradual increase in the level of regular OA exercise, such as strengthening exercises of the lower limbs. Depression restricted the performance of OA exercises in two of the eleven participants. Adaptations were made by giving extra attention to providing positive feedback, stimulating a positive attitude toward physical activities, and gradually increasing the level of physical activity.

In one participant with low vision, environmental restrictions led to adaptations in training equipment, training conditions (eg, lighting), and treatment location. No specific adaptations of the OA exercise program were needed in participants with hearing impairments (two of eleven participants).

With regard to the feasibility of the protocols, the physical therapists who tested the protocols found that they offered guidance in setting up a treatment plan/strategy, making clinical decisions, and adapting the treatment to the comorbid disease (Table 3). The following quote is from one of the physical therapists:

By using the protocol I had more knowledge about the physical capabilities of the person with OA and this specific comorbidity. Because of this I was able to design a more adequate training program and to better estimate the training intensity. This enabled me to treat the patient more intensively than I would have done without the use of the protocol.

All physical therapists indicated that the list of restrictions for exercise therapy was a conveniently arranged checklist for the diagnostic and treatment phases. The list was also helpful in the process of clinical decision making, especially when more than one comorbidity was present. If more than one comorbidity was present, more emphasis was placed on the protocol(s) for the comorbidity with the highest impact on physical functioning.

Importantly, the therapists agreed with the suggestion to increase feasibility by reducing the protocols to three main protocols. Protocol A concerned physiological adaptations (for persons with coronary artery disease, heart failure, hypertension, type 2 diabetes, obesity, and/or COPD). Protocol B concerned behavioral adaptations (for persons with chronic pain, nonspecific low back pain, and/or depression). Protocol $\mathrm{C}$ concerned environmental adaptations (for persons with visual and/or hearing impairments).

Three of eleven participants would have been excluded from treatment in the absence of the protocols. The therapists were less afraid to increase training intensity. They tailored the programs according to the individual's capacity, hereby 


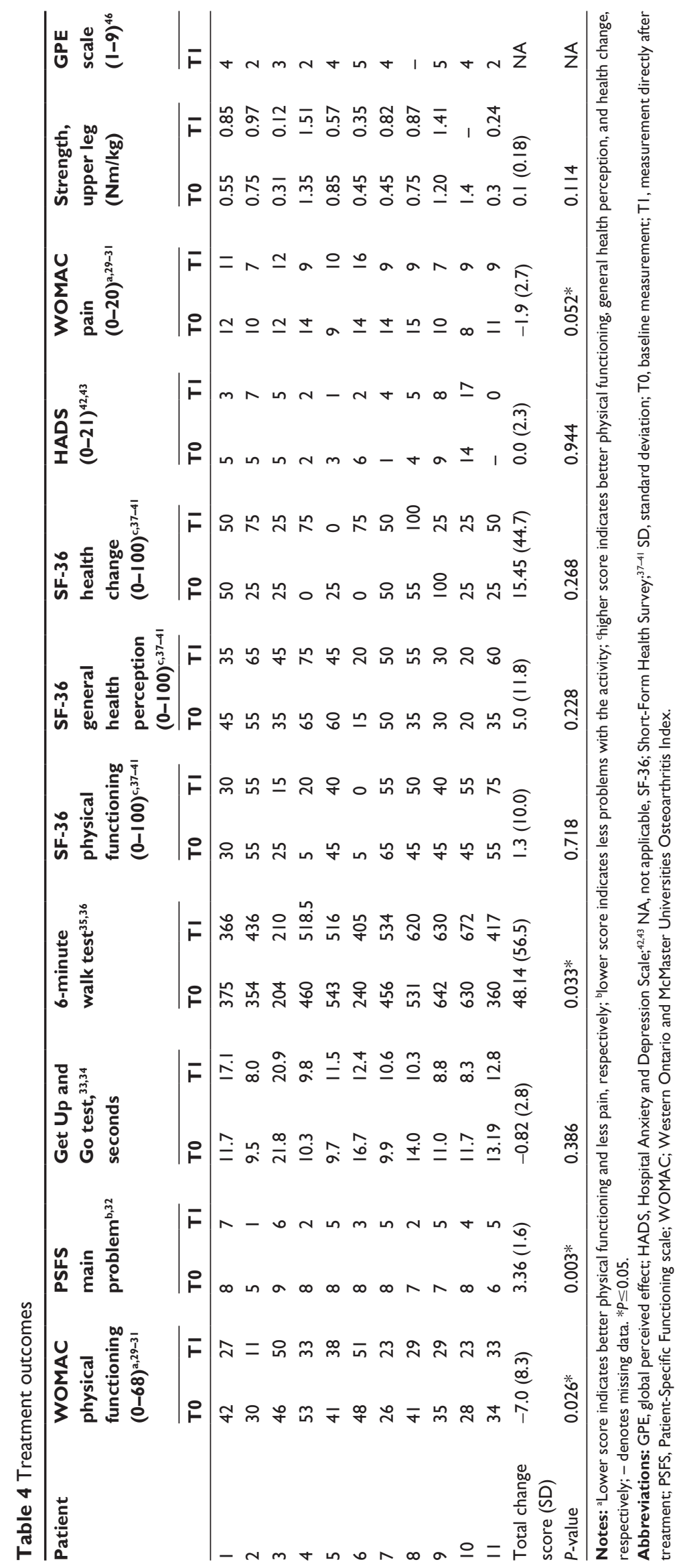


preventing adverse events. The average duration of the intake consultation was 90 minutes per patient. The protocols were feasible in persons with mild (K\&L grade 1) to severe (K\&L grade 4) OA.

All participants were satisfied with the applicability of the protocols, as indicated by a mean score of 8 points (range: 7-10) on the NRS of satisfaction. None of the participants objected to the extended duration of the intake phase. Nine of eleven participants mentioned that the therapists appeared to have a good level of knowledge about their health condition(s), which gave them more confidence in performing exercises. The following quote is from one participant:

I felt more confident in performing exercises and was less afraid to get hypoglycemia during or after the training, because the therapist had more knowledge about my diseases and training possibilities. When I was treated in primary care for my knee complaints, I dropped out in an early phase of the treatment because my knee pain was getting worse due to the high training intensity at the beginning of the program. In addition, I was afraid when feeling an increase in my heart rate during the exercises and of becoming hypoglycemic. Therefore, I wasn't really motivated to do my exercises.

Patient outcomes after treatment are presented in Table 4. On the WOMAC physical functioning scale, a statistically significant improvement $(P<0.05)$ was found, with an average increase of $18 \%$ above the baseline score. For the 6-minute walking test, the average increase was $13 \%$ above the baseline score $(P<0.05)$. There was also a statistically significant decrease in pain, as measured with the WOMAC pain subscale, where the average was $16 \%$ above the baseline score $(P<0.05)$. The main activity limitation (as ranked by each participant as being most important) on the Patient-Specific Functioning Scale questionnaire also showed a statistically significant improvement $(P<0.05)$. No significant changes were found for the other measurements. With regard to the extent to which symptoms changed over the period of treatment (Global Perceived Effect scale), four patients indicated that they were much or moderately improved after treatment; four patients reported little improvement after the treatment; and two patients reported no change in symptoms after treatment.

\section{Discussion}

Comorbidity is highly prevalent in patients with knee OA. Nevertheless, no evidence-based recommendations are available concerning comorbidity-adapted exercises in patients with knee OA. The present study concerns the development of comorbidity-adapted exercise protocols in patients with knee OA. The protocols were found to be feasible and helpful in clinical reasoning and adapting OA exercises.

To our knowledge, this is the first time that comorbidityadapted protocols have been developed for exercise therapy in patients with OA of the knee and comorbidity. Evidencebased diagnostics and treatment strategies generally overlook comorbidity. ${ }^{13,14}$ The interacting effects of diseases and their management require more complex and individualized care than simply the sum of separate guideline components.

Eleven comorbidity-adapted exercise protocols were developed for patients with knee OA and comorbidity. The protocols were found to provide guidance in clinical reasoning to direct both the diagnostic and treatment phases in persons with OA and complex, comorbidity-related health problems. The results of our field-testing revealed that the eleven protocols could be reduced to three main protocols due to overlap in diagnostics and/or treatmentrelated adaptations of the comorbidities and to improve user-friendliness. Protocol A concerned physiological adaptations (for persons with coronary artery disease, heart failure, hypertension, type 2 diabetes, obesity, and/ or COPD). Protocol B concerned behavioral adaptations (for persons with chronic pain, nonspecific low back pain, and/or depression). Protocol C concerned environmental adaptations (for persons with visual and/or hearing impairments). The protocols encourage physical therapists to think in advance about 1) how comorbidity compromises the regular application of exercise therapy by using the list of restrictions for exercise therapy of the comorbid disease and 2) how to adapt the exercise.

As expected, in participants with physiological impairments (eg, coronary disease), the training intensity and frequency and type of exercises were adapted to the comorbidity. In participants with behavioral impairments (eg, chronic pain), a combination of regular OA exercises with a behavioral approach was preferred, in which the level of physical activity was gradually increased in a time-contingent manner. In one participant with visual impairments, environmental adaptations were applied (eg, adapting the lighting in the exercise hall). Furthermore, treatment had a significant beneficial effect on physical functioning and pain. An average increase of $18 \%$ on the physical functioning subscale and a decrease of $16 \%$ on the pain subscale were found with WOMAC, which can be regarded as clinically important, relevant change. ${ }^{48}$ The treatment was safe and, by using the protocols, more patients with $\mathrm{OA}$ and comorbidity could participate in the exercise therapy. 
When taking comorbidity into account, adequate clinical reasoning is essential in order to deal with persons with a complex health status. Physical therapists need to be alert to changes in health conditions that may necessitate further adaptations of the exercises. Comorbidity may impose several different or even contradictory requirements for exercise. Physical therapists with experience in dealing with chronic conditions may have an advantage in clinical reasoning and in the adaption of exercise programs in accordance with the comorbidity. Physical therapists need to have an advanced understanding of complex system interrelationships regarding multiple morbidities. Therefore, therapists should receive specific training to increase their knowledge about various comorbidities and their effects on OA exercise therapy.

A number of remarks can be made about the usage and further development of the protocols. First, part of the results are based on personal opinions of three physical therapists working in a rehabilitation setting. To make the protocols broadly applicable, testing among various physical therapists practicing in different settings is needed. Second, the protocols were tested on eleven patients with knee OA and various comorbidities. To compare the effectiveness of the protocols to usual care, a randomized clinical trial should be performed.

\section{Conclusion}

Comorbidity-adapted exercise protocols for patients with knee OA were developed that can provide guidance in clinical reasoning with regard to diagnostics and treatment. To evaluate the effectiveness of treatment in line with our protocols, a randomized clinical trial should be performed.

\section{Acknowledgments}

The authors would like to thank MS Terbraak and DG de Rooij, $\mathrm{PhD}$, for advice and critical reading of the manuscript. In addition, we would like to thank the participants who participated in this study and the therapists for their time and contribution toward optimizing the protocols. This study was financially supported by the Royal Dutch Society for Physical Therapy.

\section{Disclosure}

The authors report no conflicts of interest in this work.

\section{References}

1. Caporali R, Cimmino MA, Sarzi-Puttini P, et al. Comorbid conditions in the AMICA study patients: effects on the quality of life and drug prescriptions by general practitioners and specialists. Semin Arthritis Rheum. 2005;35(1 Suppl 1):31-37.
2. Kadam UT, Jordan K, Croft PR. Clinical comorbidity in patients with osteoarthritis: a case-control study of general practice consulters in England and Wales. Ann Rheum Dis. 2004;63(4):408-414.

3. Feinstein AR. The pre-therapeutic classification of comorbidity in chronic disease. J Chronic Dis. 1970;23(7):455-468.

4. Reeuwijk KG, de Rooij M, van Dijk GM, Veenhof C, Steultjens MP, Dekker J. Osteoarthritis of the hip or knee: which coexisting disorders are disabling? Clin Rheumatol. 2010;29(7):739-747.

5. van Dijk GM, Veenhof C, Schellevis F, et al. Comorbidity, limitations in activities and pain in patients with osteoarthritis of the hip or knee. BMC Musculoskelet Disord. 2008;9:95.

6. van Dijk GM, Veenhof C, Spreeuwenberg P, et al; CARPA Study Group. Prognosis of limitations in activities in osteoarthritis of the hip or knee: a 3-year cohort study. Arch Phys Med Rehabil. 2010;91(1):58-66.

7. Fernandes L, Hagen KB, Bijlsma JW, et al; European League Against Rheumatism (EULAR). EULAR recommendations for the nonpharmacological core management of hip and knee osteoarthritis. Ann Rheum Dis. 2013;72(7):1125-1135.

8. Zhang W, Nuki G, Moskowitz RW, et al. OARSI recommendations for the management of hip and knee osteoarthritis: part III: changes in evidence following systematic cumulative update of research published through January 2009. Osteoarthritis Cartilage. 2010;18(4):476-499.

9. Smidt N, de Vet HC, Bouter LM, et al; Exercise Therapy Group. Effectiveness of exercise therapy: a best-evidence summary of systematic reviews. Aust J Physiother. 2005;51(2):71-85.

10. de Rooij M, Steultjens MPM, Avezaat E, et al. Restrictions and contraindications for exercise therapy in patients with hip and knee osteoarthritis and comorbidity. Phys Ther Rev. 2013;18(2):101-111.

11. Lavie CJ, Thomas RJ, Squires RW, Allison TG, Milani RV. Exercise training and cardiac rehabilitation in primary and secondary prevention of coronary heart disease. Mayo Clin Proc. 2009;84(4):373-383.

12. Myers JN, Brubaker PH. Chronic heart failure. In: Durstine JL, Moore GE, Painter PL, et al, editors. ACSM's Exercise Management for Persons with Chronic Diseases and Disabilities. 3rd ed. Champaign, IL: Human Kinetics; 2009:92-97.

13. Boyd CM, Darer J, Boult C, Fried LP, Boult L, Wu AW. Clinical practice guidelines and quality of care for older patients with multiple comorbid diseases: implications for pay for performance. JAMA. 2005;294:716-724.

14. Lugtenberg M, Burgers JS, Clancy C, Westert GP, Schneider EC. Current guidelines have limited applicability to patients with comorbid conditions: a systematic analysis of evidence-based guidelines. PLoS One. 2011;6(10):e25987.

15. Peter WF, Jansen MJ, Hurkmans EJ, et al. Guideline Steering Committee - Hip and Knee Osteoarthritis. Physiotherapy in hip and knee osteoarthritis: development of a practice guideline concerning initial assessment, treatment and evaluation. Acta Reumatol Port. 2011;36(3):268-281.Availble from http://www.fysionet-evidencebased. nl/index.php/kngf-guidelines-in-english. Accessed February 20, 2014.

16. van Weel C, Schellevis FG. Comorbidity and guidelines: conflicting interests. Lancet. 2006;367:550-551.

17. Rothstein JM, Echternach JL, Riddle DL. The Hypothesis-Oriented Algorithm for Clinicians II (HOAC II): a guide for patient management. Phys Ther. 2003;83(5):455-470.

18. Campbell M, Fitzpatrick R, Haines A, et al. Framework for design and evaluation of complex interventions to improve health. BMJ. 2000;321(7262):694-696.

19. Achttien RJ, Staal JB, Merry AHH, et al. KNGF-richtlijn hartrevalidatie [KNGF-Guideline for Cardiac Rehabilitation], The Royal Dutch Society for Physical Therapy (KNGF). 2011;121(4):1-61. URL Availble from http://www.fysionet-evidencebased.nl/images/ pdfs/guidelines_in_english/cardiac_rehabilitation_practice_ guidelines_2011.pdf. Accessed February 20, 2014.

20. Bekkering GE, Hendriks HJM, Koes BW, et al. KNGF-richtlijn lage rugpijn [KNGF-guideline low back pain], The Royal Dutch Society for Physical Therapy (KNGF). 2001;111(3). Available from Bekkering GE, Hendriks HJM, Koes BW, et al. KNGF-richtlijn lage rugpijn [KNGFguideline low back pain], The Royal Dutch Society for Physical Therapy (KNGF). 2001;111(3). Accessed February 20, 2014. 
21. Durnstine JL, Moore GE, Painter PL, et al, editors. ACSM's Exercise Management for Persons with Chronic Diseases and Disabilities. 3rd ed. Champaign, IL: Human Kinetics; 2009.

22. Gosselink RA, Langer D, Burtin C, et al. KNGF-richtlijn chronisch obstructieve longziekte [KNGF-guideline for physical therapy in chronic obstructive pulmonary disease]. Royal Dutch Society for Physical Therapy. 2008;118(4):1-60. URL Availble from http://www.fysionetevidencebased.nl/index.php/kngf-guidelines-in-english. Accessed February 20, 2014.

23. Expert Committee on the Diagnosis and Classification of Diabetes Mellitus. Report of the expert committee on the diagnosis and classification of diabetes mellitus. Diabetes Care. 2003;26 Suppl 1:S5-S20.

24. van Marwijk HWJ, Grundmeijer HGLM, van Gelderen MG, et al. NHG-Standaard Depressieve stoornis (depressie) [NHG Standard depressive disorder (depression)]. Huisarts Wet. 2003;46(11):614-633. Dutch.

25. Walma EP, Thomas S, Prins A, Grundmeijer HGLM, Laan van der JR, Wiersma TJ. NHG-Standaard Hypertensie [NHG Standard hypertension]. Huisarts Wet. 2003;46:435-449. Dutch.

26. Handbook for Good Clinical Research Practice of the World Health Organization, and Declaration of Helsinki principles. Available from: http://www.wma.net/en/30publications/10policies/b3/. Accessed February 20, 2014.

27. Altman R, Asch E, Bloch D, et al. Development of criteria for the classification and reporting of osteoarthritis. Classification of osteoarthritis of the knee. Diagnostic and Therapeutic Criteria Committee of the American Rheumatism Association. Arthritis Rheum. 1986;29(8): 1039-1049.

28. Miller MD, Paradis CF, Houck PR, et al. Rating chronic medical illness burden in geropsychiatric practice and research: application of the Cumulative Illness Rating Scale. Psychiatry Res. 1992;41(3):237-248.

29. Bellamy N, Buchanan WW, Goldsmith CH, Campbell J, Stitt LW. Validation study of WOMAC: a health status instrument for measuring clinically important patient relevant outcomes to antirheumatic drug therapy in patients with osteoarthritis of the hip or knee. J Rheumatol. 1988;15(12):1833-1840.

30. Roorda LD, Jones CA, Waltz M, et al. Satisfactory cross cultural equivalence of the Dutch WOMAC in patients with hip osteoarthritis waiting for arthroplasty. Ann Rheum Dis. 2004;63(1):36-42.

31. Veenhof C, Bijlsma JW, van den Ende CH, van Dijk GM, Pisters MF, Dekker J. Psychometric evaluation of osteoarthritis questionnaires: a systematic review of the literature. Arthritis Rheum. 2006;55: 480-492.

32. Westaway MD, Stratford PW, Binkley JM. The patient-specific functional scale: validation of its use in persons with neck dysfunction. J Orthop Sports Phys Ther. 1998;27(5):331-338.

33. Hurley MV, Scott DL, Rees J, Newham DJ. Sensorimotor changes and functional performance in patients with knee osteoarthritis. Ann Rheum Dis. 1997;56:641-648.

34. van der Esch M, Steultjens M, Harlaar J, Wolterbeek N, Knol D, Dekker J. Varus-valgus motion and functional ability in patients with knee osteoarthritis. Ann Rheum Dis. 2008;67:471-477.
35. Stratford PW, Kennedy DM, Woodhouse LJ. Performance measures provide assessments of pain and function in people with advanced osteoarthritis of the hip or knee. Phys Ther. 2006;86(11):1489-1496.

36. Steffen TM, Hacker TA, Mollinger L. Age- and gender-related test performance in community-dwelling elderly people: Six-Minute Walk Test, Berg Balance Scale, Timed Up and Go Test, and gait speeds. Phys Ther. 2002;82(2):128-137.

37. Ware JE Jr, Sherbourne CD. The MOS 36-item short-form health survey (SF-36). I. Conceptual framework and item selection. Med Care. 1992;30(6):473-483.

38. McHorney CA, Ware JE Jr, Raczek AE. The MOS 36-Item ShortForm Health Survey (SF-36): II. Psychometric and clinical tests of validity in measuring physical and mental health constructs. Med Care. 1993;31:247-263.

39. McHorney CA, Ware JE Jr, Lu JF, Sherbourne CD. The MOS 36-item ShortForm Health Survey (SF-36): III. Tests of data quality, scaling assumptions, and reliability across diverse patients groups. Med Care. 1994;32:40-66.

40. Brazier JE, Harper R, Jones NM, et al. Validating the SF-36 health survey questionnaire: new outcome measure for primary care. BMJ. 1992;305:160-164.

41. Lyons RA, Perry HM, Littlepage BN. Evidence for the validity of the Short-form 36 Questionnaire (SF-36) in an elderly population. Age Ageing. 1994;23:182-184.

42. Zigmond AS, Snaith RP. The hospital anxiety and depression scale. Acta Psychiatr Scand. 1983;67(6):361-370.

43. Axford J, Butt A, Heron C, et al. Prevalence of anxiety and depression in osteoarthritis: use of the hospital anxiety and depression scale as a screening tool. Clin Rheumatol. 2010;29:1277-1283.

44. van der Esch M, Steultjens M, Harlaar J, Knol D, Lems W, Dekker J. Joint proprioception, muscle strength, and functional ability in patients with osteoarthritis of the knee. Arthritis Rheum. 2007;57(5):787-793.

45. Kean CO, Birmingham TB, Garland SJ, Bryant DM, Giffin JR. Minimal detectable change in quadriceps strength and voluntary muscle activation in patients with knee osteoarthritis. Arch Phys Med Rehabil. 2010;91:1447-1451.

46. Kamper SJ, Ostelo RW, Knol DL, Maher CG, de Vet HC, Hancock MJ. Global Perceived Effect scales provided reliable assessments of health transition in people with musculoskeletal disorders, but ratings are strongly influenced by current status. J Clin Epidemiol. 2010;63:760-766.

47. Kellgren JH, Lawrence JS. Radiological assessment of osteo-arthrosis. Ann Rheum Dis. 1957;16(4):494-502.

48. Angst F, Aeschlimann A, Stucki G. Smallest detectable and minimal clinically important differences of rehabilitation intervention with their implications for required sample sizes using WOMAC and SF-36 quality of life measurement instruments in patients with osteoarthritis of the lower extremities. Arthritis Rheum. 2001;45(4):384-391.

49. Veenhof C, Köke AJ, Dekker J, et al. Effectiveness of behavioral graded activity in patients with osteoarthritis of the hip and/or knee: a randomized clinical trial. Arthritis Rheum. 2006;55(6):925-934.

50. Borg GAV. Psychophysical bases of perceived exertion. Med Sci Sports Exerc. 1982;14(5):377-381.
Clinical Interventions in Aging

\section{Publish your work in this journal}

Clinical Interventions in Aging is an international, peer-reviewed journal focusing on evidence-based reports on the value or lack thereof of treatments intended to prevent or delay the onset of maladaptive correlates of aging in human beings. This journal is indexed on PubMed Central, MedLine, the American Chemical Society's 'Chemical Abstracts
Dovepress

Service' (CAS), Scopus and the Elsevier Bibliographic databases. The manuscript management system is completely online and includes a very quick and fair peer-review system, which is all easy to use. Visit $\mathrm{http}: / /$ www.dovepress.com/testimonials.php to read real quotes from published authors. 